\title{
On the odd part of tame kernels of biquadratic number fields
}

by

\author{
HAIYAN ZHOU (Nanjing)
}

1. Introduction. Let $F$ be an algebraic number field, $\mathcal{O}_{F}$ the ring of integers in $F$, and $K_{2}$ the Milnor $K$-functor. For a quadratic number field, the 2-primary part of $K_{2} \mathcal{O}_{F}$ has been intensively studied (see [5]-[10]). For an odd prime $p$, it is interesting to study the $p$-primary part of the tame kernels of number fields; some results have been found in [1]-[4].

In this paper we investigate the odd part of the tame kernel $K_{2} \mathcal{O}_{F}$ for a biquadratic field $F$, where $F=\mathbb{Q}\left(\sqrt{d_{1}}, \sqrt{d_{2}}\right)$. Section 2 studies the $p$-Sylow subgroup of the class group of the cyclotomic extension of $F$. Let $p$ be a regular prime. In Section 3, we prove some results connecting the $p$-rank of the tame kernel $K_{2} \mathcal{O}_{F}$ with the $p$-rank of the class groups of some subfields of the number field $F\left(\zeta_{p}\right)$. In particular, when $p=3,5$, we get some results connecting the $p$-rank of $K_{2} \mathcal{O}_{F}$ with the $p$-rank of the class groups of some quadratic fields. As an application, we calculate the 3-rank of the tame kernels $K_{2} \mathcal{O}_{F}$ when $F=\mathbb{Q}\left(\sqrt{d_{1}}, \sqrt{d_{2}}\right)$, where $-50<d_{1}, d_{2}<50$. For any odd prime $p$ and a positive integer $n \geq 2$, in Section 4 , we prove explicitly some relations between the groups $\left(\mu_{p^{n}} \otimes C l\left(\mathcal{O}_{F\left(\zeta_{p^{n}}\right)}[1 / p]\right)\right)_{\operatorname{Gal}\left(F\left(\zeta_{p^{n}}\right) / F\right)}$ and $K_{2} \mathcal{O}_{F} / p^{n}$ by using a map of Keune.

2. Biquadratic number fields. In this section, we give more information on cyclotomic extensions of biquadratic fields. We fix the following notation.

Let $F=\mathbb{Q}\left(\sqrt{d_{1}}, \sqrt{d_{2}}\right), F_{1}=\mathbb{Q}\left(\sqrt{d_{1}}\right), F_{2}=\mathbb{Q}\left(\sqrt{d_{2}}\right)$, with $d_{1}$ and $d_{2}$ squarefree. We assume that $\left(d_{1}, d_{2}\right)=1, p$ is an odd prime, $n$ a positive integer, $\zeta_{p^{n}}$ a primitive root of unity of degree $p^{n}, L=F\left(\zeta_{p^{n}}\right), G=\operatorname{Gal}(L / F)$. Clearly, $G$ is cyclic as a subgroup of the cyclic group $\operatorname{Gal}\left(\mathbb{Q}\left(\zeta_{p^{n}}\right) / \mathbb{Q}\right)$. Let $\mathfrak{p}$ be a prime ideal of $F$ dividing $p$, and let $Z_{\mathfrak{p}}$ be the decomposition group of $\mathfrak{p}$ in the abelian extension $L / F$. Denote by $e_{\mathfrak{p}}$ the ramification degree of 
$\mathfrak{p}$ in $L / F$. For a number field $*$, we denote by $A_{*}$ and $A_{*}[1 / p]$ the $p$-Sylow subgroups of $C l\left(\mathcal{O}_{*}\right)$ and $C l\left(\mathcal{O}_{*}[1 / p]\right)$ respectively.

We consider the following cases.

1) $d_{1}=p^{*}=(-1)^{(p-1) / 2} p$. Then $F_{1}$ is the unique quadratic subfield of $\mathbb{Q}\left(\zeta_{p^{n}}\right)$, and $p$ is totally ramified in $F_{1}$. By the assumption, $p \nmid d_{2}$. We consider two subcases.

a) $\left(\frac{d_{2}}{p}\right)=1$. Then $p$ splits in $F_{2}$. It follows that $p \mathcal{O}_{F}=\mathfrak{p}_{1}^{2} \mathfrak{p}_{2}^{2}$. Since $p$ is totally ramified in $\mathbb{Q}\left(\zeta_{p^{n}}\right)$, the prime ideals $\mathfrak{p}_{1}$ and $\mathfrak{p}_{2}$ of $F$ are totally ramified in $L / F$, and $e_{\mathfrak{p}_{1}}=e_{\mathfrak{p}_{2}}=(L: F)$ and $Z_{\mathfrak{p}_{1}}=Z_{\mathfrak{p}_{2}}=G$.

b) $\left(\frac{d_{2}}{p}\right)=-1$. Then $p$ is inert in $F_{2}$, hence $p \mathcal{O}_{F}=\mathfrak{p}^{2}$. Since $p$ is totally ramified in $\mathbb{Q}\left(\zeta_{p^{n}}\right)$, the prime ideal $\mathfrak{p}$ of $F$ is totally ramified in $L / F$ with $e_{\mathfrak{p}}=(L: F)$ and $Z_{\mathfrak{p}}=G$.

2) $d_{1} \neq p^{*}$ and $p \mid d_{1}$. Then $p$ is totally ramified in $F_{1}$. Let $d_{1}=p^{*} d_{1}^{\prime}$. By the assumption, $p \nmid d_{2}$. We consider four subcases.

a) $\left(\frac{d_{1}^{\prime}}{p}\right)=1$ and $\left(\frac{d_{2}}{p}\right)=1$. Then $p$ splits in $\mathbb{Q}\left(\sqrt{d_{1}^{\prime}}\right)$ and $F_{2}$, so $p$ splits in $\mathbb{Q}\left(\sqrt{d_{1}^{\prime}}, \sqrt{d_{2}}\right)$ and $p \mathcal{O}_{F}=\mathfrak{p}_{1}^{2} \mathfrak{p}_{2}^{2}$. Let $E=\mathbb{Q}\left(\sqrt{d_{1}}, \sqrt{d_{2}}, \sqrt{p^{*}}\right)=$ $\mathbb{Q}\left(\sqrt{d_{1}^{\prime}}, \sqrt{d_{2}}, \sqrt{p^{*}}\right)$. Then the prime ideals $\mathfrak{p}_{1}$ and $\mathfrak{p}_{2}$ split in $E / F$. Since $p$ is totally ramified in $\mathbb{Q}\left(\zeta_{p^{n}}\right)$, we have $e_{\mathfrak{p}_{1}}=e_{\mathfrak{p}_{2}}=(L: E)=(L: F) / 2$. The decomposition fields of $\mathfrak{p}_{1}$ and $\mathfrak{p}_{2}$ in $L / F$ are both $E$, and $Z_{\mathfrak{p}_{1}}=Z_{\mathfrak{p}_{2}}=$ $\operatorname{Gal}(L / E)=\operatorname{Gal}\left(\mathbb{Q}\left(\zeta_{p^{n}}\right) / \mathbb{Q}\left(\sqrt{p^{*}}\right)\right)$.

b) $\left(\frac{d_{1}^{\prime}}{p}\right)=1$ and $\left(\frac{d_{2}}{p}\right)=-1$. Then $p$ splits in $\mathbb{Q}\left(\sqrt{d_{1}^{\prime}}\right)$ and is inert in $F_{2}$, so $p \mathcal{O}_{F}=\mathfrak{p}^{2}$. Hence the prime ideal $\mathfrak{p}$ splits in $E / F$. Since $p$ is totally ramified in $\mathbb{Q}\left(\zeta_{p^{n}}\right)$, the ramification degree $e_{\mathfrak{p}}$ equals $(L: E)=(L: F) / 2$. The decomposition field of $\mathfrak{p}$ in $L / F$ is $E$ and $Z_{\mathfrak{p}}=\operatorname{Gal}(L / E)=\operatorname{Gal}\left(\mathbb{Q}\left(\zeta_{p^{n}}\right) / \mathbb{Q}\left(\sqrt{p^{*}}\right)\right)$.

c) $\left(\frac{d_{1}^{\prime}}{p}\right)=-1$ and $\left(\frac{d_{2}}{p}\right)=1$. Then $p$ splits in $F_{2}$ and is inert in $\mathbb{Q}\left(\sqrt{d_{1}^{\prime}}\right)$, so $p \mathcal{O}_{F}=\mathfrak{p}_{1}^{2} \mathfrak{p}_{2}^{2}$. Hence the prime ideals $\mathfrak{p}_{1}$ and $\mathfrak{p}_{2}$ are inert in $E / F$. Since $p$ is totally ramified in $\mathbb{Q}\left(\zeta_{p^{n}}\right)$, we have $e_{\mathfrak{p}_{1}}=e_{\mathfrak{p}_{2}}=(L: E)=(L: F) / 2$. The decomposition fields of $\mathfrak{p}_{1}$ and $\mathfrak{p}_{2}$ in $L / F$ are both $F$, and $Z_{\mathfrak{p}_{1}}=Z_{\mathfrak{p}_{2}}=$ $\operatorname{Gal}(L / F)=\operatorname{Gal}\left(\mathbb{Q}\left(\zeta_{p^{n}}\right) / \mathbb{Q}\right)$.

d) $\left(\frac{d_{1}^{\prime}}{p}\right)=-1$ and $\left(\frac{d_{2}}{p}\right)=-1$. Then $p$ is inert in $\mathbb{Q}\left(\sqrt{d_{1}^{\prime}}\right)$ and $F_{2}$, so $p \mathcal{O}_{F}=\mathfrak{p}^{2}$. Since $\left(\frac{d_{1}^{\prime} d_{2}}{p}\right)=1, p$ splits in $\mathbb{Q}\left(\sqrt{d_{1}^{\prime} d_{2}}\right)$. Hence the prime ideal $\mathfrak{p}$ splits in $E / F$. Since $p$ is totally ramified in $\mathbb{Q}\left(\zeta_{p^{n}}\right)$, we have $e_{\mathfrak{p}}=(L: E)=$ $(L: F) / 2$. The decomposition field of $\mathfrak{p}$ in $L / F$ is $E$, and $Z_{\mathfrak{p}}=\operatorname{Gal}(L / E)=$ $\operatorname{Gal}\left(\mathbb{Q}\left(\zeta_{p^{n}}\right) / \mathbb{Q}\left(\sqrt{p^{*}}\right)\right)$.

3) $p \nmid d_{1}$ and $p \nmid d_{2}$. Then $p$ is unramified in $F$. Since $p$ is totally ramified in $\mathbb{Q}\left(\zeta_{p^{n}}\right)$, it is totally ramified in $L / F$. Hence the ramification degree $e_{\mathfrak{p}}$ of the prime ideal $\mathfrak{p} \mid p$ of $F$ is $(L: F)$. The decomposition field of $\mathfrak{p}$ in $L / F$ is $F$, and $Z_{\mathfrak{p}}=\operatorname{Gal}(L / F)=\operatorname{Gal}\left(\mathbb{Q}\left(\zeta_{p^{n}}\right) / \mathbb{Q}\right)$. 
From the above conclusions, we deduce the following lemma:

Lemma 2.1. Let $L=F\left(\zeta_{p}\right)$. Assume that $S_{F}^{\prime}$ is the set of prime ideals of $F$ which divide $p$ and split completely in $L$. Then:

(i) $\left|S_{F}^{\prime}\right|=2$ if $p=3, d_{1} \equiv 3(\bmod 9)$ and $d_{2} \equiv 1(\bmod 3)$,

(ii) $\left|S_{F}^{\prime}\right|=1$ if $p=3,3 \mid d_{1}$ and $d_{2} \equiv 2(\bmod 3)$,

(iii) $\left|S_{F}^{\prime}\right|=0$ otherwise.

LEMMA 2.2. Assume that $p$ is an odd prime and $n$ a positive integer. Let $F=\mathbb{Q}\left(\sqrt{d_{1}}, \sqrt{d_{2}}\right), K=\mathbb{Q}\left(\zeta_{p^{n}}+\zeta_{p^{n}}^{-1}\right), L=F\left(\zeta_{p^{n}}\right)$. Let $L_{1}=K\left(\sqrt{d_{1}}\right)$, $L_{2}=K\left(\sqrt{d_{1} d_{2}}\right), L_{3}=K\left(\left(\zeta_{p^{n}}-\zeta_{p^{n}}^{-1}\right) \sqrt{d_{1} d_{2}}\right), L_{4}=K\left(\sqrt{d_{2}}\right), L_{5}=K\left(\left(\zeta_{p^{n}}-\right.\right.$ $\left.\left.\zeta_{p^{n}}^{-1}\right) \sqrt{d_{2}}\right), L_{6}=K\left(\left(\zeta_{p^{n}}-\zeta_{p^{n}}^{-1}\right) \sqrt{d_{1}}\right), L_{7}=K\left(\zeta_{p^{n}}-\zeta_{p^{n}}^{-1}\right)$. Assume further that $p$ is regular and $F \cap \mathbb{Q}\left(\zeta_{p^{n}}\right)=\mathbb{Q}$. Then

$$
A_{L}=A_{L_{1}} \oplus A_{L_{2}} \oplus A_{L_{3}} \oplus A_{L_{4}} \oplus A_{L_{5}} \oplus A_{L_{6}} .
$$

Proof. Let $G=\operatorname{Gal}(L / K)$, so we have $\tau_{1}, \tau_{2}, \sigma \in G$ such that

$$
\begin{aligned}
& \tau_{1}: \sqrt{d_{1}} \mapsto-\sqrt{d_{1}}, \sqrt{d_{2}} \mapsto \sqrt{d_{2}}, \zeta_{p^{n}} \mapsto \zeta_{p^{n}}, \\
& \tau_{2}: \sqrt{d_{1}} \mapsto \sqrt{d_{1}}, \sqrt{d_{2}} \mapsto-\sqrt{d_{2}}, \zeta_{p^{n}} \mapsto \zeta_{p^{n}}, \\
& \sigma: \sqrt{d_{1}} \mapsto \sqrt{d_{1}}, \sqrt{d_{2}} \mapsto \sqrt{d_{2}}, \zeta_{p^{n}} \mapsto \zeta_{p^{n}}^{-1} .
\end{aligned}
$$

Also, $\left\langle\sigma, \tau_{2}\right\rangle=\operatorname{Gal}\left(L / L_{1}\right),\left\langle\sigma, \tau_{1} \tau_{2}\right\rangle=\operatorname{Gal}\left(L / L_{2}\right),\left\langle\sigma \tau_{1}, \tau_{1} \tau_{2}\right\rangle=\operatorname{Gal}\left(L / L_{3}\right)$, $\left\langle\sigma, \tau_{1}\right\rangle=\operatorname{Gal}\left(L / L_{4}\right),\left\langle\sigma \tau_{2}, \tau_{1}\right\rangle=\operatorname{Gal}\left(L / L_{5}\right),\left\langle\sigma \tau_{1}, \tau_{2}\right\rangle=\operatorname{Gal}\left(L / L_{6}\right),\left\langle\tau_{1}, \tau_{2}\right\rangle$ $=\operatorname{Gal}\left(L / L_{7}\right)$. Then we have idempotents in $\mathbb{Z}_{p}[G]$ :

$$
\begin{array}{ll}
\eta_{0}=\left(\frac{1+\tau_{1}}{2}\right)\left(\frac{1+\tau_{2}}{2}\right)\left(\frac{1+\sigma}{2}\right), & \eta_{1}=\left(\frac{1-\tau_{1}}{2}\right)\left(\frac{1+\tau_{2}}{2}\right)\left(\frac{1+\sigma}{2}\right), \\
\eta_{2}=\left(\frac{1-\tau_{1}}{2}\right)\left(\frac{1-\tau_{2}}{2}\right)\left(\frac{1+\sigma}{2}\right), & \eta_{3}=\left(\frac{1-\tau_{1}}{2}\right)\left(\frac{1-\tau_{2}}{2}\right)\left(\frac{1-\sigma}{2}\right), \\
\eta_{4}=\left(\frac{1+\tau_{1}}{2}\right)\left(\frac{1-\tau_{2}}{2}\right)\left(\frac{1+\sigma}{2}\right), & \eta_{5}=\left(\frac{1+\tau_{1}}{2}\right)\left(\frac{1-\tau_{2}}{2}\right)\left(\frac{1-\sigma}{2}\right), \\
\eta_{6}=\left(\frac{1-\tau_{1}}{2}\right)\left(\frac{1+\tau_{2}}{2}\right)\left(\frac{1-\sigma}{2}\right), & \eta_{7}=\left(\frac{1+\tau_{1}}{2}\right)\left(\frac{1+\tau_{2}}{2}\right)\left(\frac{1-\sigma}{2}\right) .
\end{array}
$$

It is easy to verify $1=\eta_{0}+\eta_{1}+\eta_{2}+\eta_{3}+\eta_{4}+\eta_{5}+\eta_{6}+\eta_{7}$.

We have $\eta_{0} A_{L} \subseteq N_{L / K} A_{L}=0$ since $p$ is regular. Furthermore, $\eta_{1} A_{L}=$ $\frac{1}{8}\left(1-\tau_{1}\right) N_{L / L_{1}} A_{L}$, so $\eta_{1} A_{L} \subseteq A_{L_{1}}$. On the other hand, for any $a \in A_{L_{1}}$, we have $\sigma a=a, \tau_{2} a=a,\left(1+\tau_{1}\right) a=0$, i.e., $\tau_{1} a=-a$, since $1+\tau_{1}=N_{L_{1} / K}$ and $p$ is regular. Hence, $\eta_{1} a=\frac{1}{8}\left(1-\tau_{1}\right)\left(1+\tau_{2}\right)(1+\sigma) a=a$, and so $\eta_{1} A_{L}=A_{L_{1}}$. Similarly, $\eta_{4} A_{L}=A_{L_{4}}$ and $\eta_{7} A_{L}=A_{L_{7}}$. 
Also,

$$
\begin{aligned}
& \eta_{2}=\left(\frac{1-\tau_{1}}{2}\right)\left(\frac{1-\tau_{2}}{2}\right)\left(\frac{1+\sigma}{2}\right)=\frac{1}{8}\left(1-\tau_{1}\right)\left(1+\tau_{1} \tau_{2}\right)(1+\sigma), \\
& \eta_{3}=\left(\frac{1-\tau_{1}}{2}\right)\left(\frac{1-\tau_{2}}{2}\right)\left(\frac{1-\sigma}{2}\right)=\frac{1}{8}\left(1-\tau_{1}\right)\left(1+\tau_{1} \tau_{2}\right)\left(1+\sigma \tau_{1}\right), \\
& \eta_{5}=\left(\frac{1+\tau_{1}}{2}\right)\left(\frac{1-\tau_{2}}{2}\right)\left(\frac{1-\sigma}{2}\right)=\frac{1}{8}\left(1-\tau_{2}\right)\left(1+\tau_{1}\right)\left(1+\sigma \tau_{2}\right), \\
& \eta_{6}=\left(\frac{1-\tau_{1}}{2}\right)\left(\frac{1+\tau_{2}}{2}\right)\left(\frac{1-\sigma}{2}\right)=\frac{1}{8}\left(1-\tau_{1}\right)\left(1+\tau_{2}\right)\left(1+\sigma \tau_{1}\right) .
\end{aligned}
$$

By the above proof, $\eta_{i} A_{L}=A_{L_{i}}, i=2,3,5,6$. Since $p$ is regular, we have $A_{L_{7}}=0$, and the conclusion follows.

3. $p$-rank. In the following, we assume that $F \cap \mathbb{Q}\left(\zeta_{p}\right)=\mathbb{Q}$. Let $L=$ $F\left(\zeta_{p}\right)$ with $\Gamma=\operatorname{Gal}(L / \mathbb{Q}) \cong G \times T$, where $G=\operatorname{Gal}\left(\mathbb{Q}\left(\zeta_{p}\right) / \mathbb{Q}\right)$ and $T=$ $\operatorname{Gal}(F / \mathbb{Q})$.

Let $\omega$ be the Teichmüller character of the group $(\mathbb{Z} / p)^{*}$. We fix a primitive root $g(\bmod p)$ and let $\sigma:=\sigma_{g}\left(\sigma_{g}\left(\zeta_{p}\right)=\zeta_{p}^{g}\right)$. We have the following idempotents:

$$
\varepsilon_{j}=\frac{1}{p-1} \sum_{a=1}^{p-1} \omega^{j}(a) \sigma_{a}^{-1}=\frac{1}{p-1} \sum_{k=0}^{p-2} \omega^{j k}(g) \sigma^{-k}, \quad 0 \leq j \leq p-2 .
$$

Let $\lambda: C l\left(\mathcal{O}_{L}\right) \rightarrow C l\left(\mathcal{O}_{L}[1 / p]\right)$ be the homomorphism induced by the imbedding $\mathcal{O}_{L} \rightarrow \mathcal{O}_{L}[1 / p]$. Evidently $\lambda$ is a surjective homomorphism of $\Gamma$-modules.

Let $E$ be the maximal unramified $p$-extension of the field $L$ with an elementary abelian Galois group $H:=\operatorname{Gal}(E / L)$. Since $\mu_{p}:=\left\langle\zeta_{p}\right\rangle \subset L$, $E / L$ is a Kummer extension, i.e., $E=L\left(B^{1 / p}\right)$, for some subgroup $B$ of $L^{*}$ containing $L^{* p}$. Let $B_{0}:=B / L^{* p}$. For every $b \in L^{*}$ and $b_{0}=b L^{* p}$, we have $b_{0} \in B_{0}$ iff $b$ is singular primary, i.e., $(b)=\mathfrak{a}^{p}$ for some ideal $\mathfrak{a}$ of $L$, and $x^{p} \equiv b\left(\bmod p\left(1-\zeta_{p}\right)\right)$ for some $x \in L^{*}$. Consequently, we have a homomorphism of $\Gamma$-modules:

$$
\phi: B_{0} \rightarrow\left(A_{L}\right)_{p}, \quad \phi\left(b_{0}\right)=C l(\mathfrak{a}) .
$$

Let $U_{L}^{\prime}$ be the group of singular primary units of $L$. Then $\operatorname{Ker} \phi \cong U_{L}^{\prime} / U_{L}^{p}$ (see $[2]$ ).

Lemma 3.1 ([2]). Let $F$ be a number field with $F \cap \mathbb{Q}\left(\zeta_{p}\right)=\mathbb{Q}$, and let $p$ be an odd prime. Then

$$
p-\operatorname{rank}\left(K_{2} \mathcal{O}_{F}\right)=p-\operatorname{rank}\left(\varepsilon_{p-2} A_{L}[1 / p]\right)+\left|S_{F}^{\prime}\right| .
$$


Lemma 3.2. The mapping $\lambda: \varepsilon_{p-2} C l\left(\mathcal{O}_{L}\right) \rightarrow \varepsilon_{p-2} C l\left(\mathcal{O}_{L}[1 / p]\right)$ is an isomorphism if one of the following conditions is satisfied:

1) $p \nmid d_{1}$ and $p \nmid d_{2}$,

2) $d_{1}=p d_{1}^{\prime},\left(\frac{d_{1}^{\prime}}{p}\right)=-1$ and $\left(\frac{d_{2}}{p}\right)=1$,

3) $p \neq 3, p \mid d_{1}$ and $\left(\frac{d_{2}}{p}\right)=-1$.

Proof. Let $\mathfrak{P}$ be the unique prime ideal over $p$ in $L$. The group $\operatorname{Ker} \lambda$ is generated by the class containing the prime ideal $\mathfrak{P}$ of $L$. If 1 ) and 2) are satisfied, then the prime ideal $\mathfrak{p} \mid p$ of $F$ does not split in $L$ by 1) and 3) of Section 2. So we have $\sigma(\mathfrak{P})=\mathfrak{P}$. Therefore, $\mathfrak{P} \in \varepsilon_{0} C l\left(\mathcal{O}_{L}\right)$.

Suppose now condition 3) is satisfied. Then by $2 \mathrm{~b}$ ) and $2 \mathrm{~d}$ ) of Section 2, in $L$ we have $\left(1-\zeta_{p}\right)=\mathfrak{P}_{1} \mathfrak{P}_{2}$, where $\mathfrak{P}_{1}$ and $\mathfrak{P}_{2}$ are different prime ideals of $E$. Consequently $\sigma\left(\mathfrak{P}_{1}\right)=\mathfrak{P}_{1}$ or $\mathfrak{P}_{2}$. Thus for $a=C l\left(\mathfrak{P}_{1}\right)$ we have $\sigma(a)= \pm a$. Evidently $a$ generates Ker $\lambda$. Assume that $a^{m} \in \varepsilon_{p-2} C l\left(\mathcal{O}_{L}\right)$ for some $m$. Then

$$
\begin{aligned}
a^{m} & =\varepsilon_{p-2} a^{m}=\frac{1}{p-1} \sum_{k=0}^{p-2} \omega^{k(p-2)}(g) \sigma^{-k} a^{m} \\
& =\frac{1}{p-1} \sum_{k=0}^{p-2} \omega^{k(p-2)}(g)( \pm 1)^{k m} a^{m} \\
& =\frac{1}{p-1} \frac{1-\left(\omega^{p-2}(g)( \pm 1)^{m}\right)^{p-1}}{1-\omega^{p-2}(g)( \pm 1)^{m}} a^{m} .
\end{aligned}
$$

Since $p \neq 3, p-2 \neq \frac{1}{2}(p-1)$. It follows that $\omega^{p-2}(g)( \pm 1)^{m} \neq 1$. Therefore $a^{m}=0$. This completes the proof.

Theorem 3.3. Assume that $p$ is a regular prime. Let $K=\mathbb{Q}\left(\zeta_{p}+\zeta_{p}^{-1}\right)$, $L=F\left(\zeta_{p}\right), L_{1}=K\left(\sqrt{d_{1}}\right), L_{2}=K\left(\sqrt{d_{1} d_{2}}\right), L_{3}=K\left(\left(\zeta_{p}-\zeta_{p}^{-1}\right) \sqrt{d_{1} d_{2}}\right)$, $L_{4}=K\left(\sqrt{d_{2}}\right), L_{5}=K\left(\left(\zeta_{p}-\zeta_{p}^{-1}\right) \sqrt{d_{2}}\right), L_{6}=K\left(\left(\zeta_{p}-\zeta_{p}^{-1}\right) \sqrt{d_{1}}\right)$.

(i) If $p>3$, then

$$
\begin{aligned}
p-\operatorname{rank}\left(K_{2} \mathcal{O}_{F}\right)= & p-\operatorname{rank}\left(\varepsilon_{p-2} A_{L_{3}}[1 / p]\right)+p-\operatorname{rank}\left(\varepsilon_{p-2} A_{L_{5}}[1 / p]\right) \\
& +p-\operatorname{rank}\left(\varepsilon_{p-2} A_{L_{6}}[1 / p]\right)
\end{aligned}
$$

provided $d_{1}=p d_{1}^{\prime},\left(\frac{d_{1}^{\prime}}{p}\right)=1$ and $\left(\frac{d_{2}}{p}\right)=1$, while

$$
\begin{aligned}
p-\operatorname{rank}\left(K_{2} \mathcal{O}_{F}\right)= & p-\operatorname{rank}\left(\varepsilon_{p-2} A_{L_{3}}\right)+p-\operatorname{rank}\left(\varepsilon_{p-2} A_{L_{5}}\right) \\
& +p-\operatorname{rank}\left(\varepsilon_{p-2} A_{L_{6}}\right)
\end{aligned}
$$

otherwise. 
(ii) If $3 \nmid d_{1}$ and $3 \nmid d_{2}$, or $d_{1} \equiv 6(\bmod 9)$ and $d_{2} \equiv 1(\bmod 3)$, then $3-\operatorname{rank}\left(K_{2} \mathcal{O}_{F}\right)=3-\operatorname{rank}\left(A_{L_{3}}\right)+3-\operatorname{rank}\left(A_{L_{5}}\right)+3-\operatorname{rank}\left(A_{L_{6}}\right)$, where $L_{3}=\mathbb{Q}\left(\sqrt{-3 d_{1} d_{2}}\right), L_{5}=\mathbb{Q}\left(\sqrt{-3 d_{2}}\right), L_{6}=\mathbb{Q}\left(\sqrt{-3 d_{1}}\right)$.

(iii) If $d_{1} \equiv 3(\bmod 9)$ and $d_{2} \equiv 1(\bmod 3)$, then $3-\operatorname{rank}\left(K_{2} \mathcal{O}_{F}\right)=3-\operatorname{rank}\left(A_{L_{3}}\right)+3-\operatorname{rank}\left(A_{L_{5}}\right)+3-\operatorname{rank}\left(A_{L_{6}}\right)+2$, where $L_{3}=\mathbb{Q}\left(\sqrt{-3 d_{1} d_{2}}\right), L_{5}=\mathbb{Q}\left(\sqrt{-3 d_{2}}\right), L_{6}=\mathbb{Q}\left(\sqrt{-3 d_{1}}\right)$.

(iv) If $d_{1} \equiv 6(\bmod 9)$ and $d_{2} \equiv 2(\bmod 3)$, then $3-\operatorname{rank}\left(K_{2} \mathcal{O}_{F}\right)=3-\operatorname{rank}\left(A_{L_{3}}\right)+3-\operatorname{rank}\left(A_{L_{5}}\right)+3-\operatorname{rank}\left(A_{L_{6}}\right)+1$, where $L_{3}=\mathbb{Q}\left(\sqrt{-3 d_{1} d_{2}}\right), L_{5}=\mathbb{Q}\left(\sqrt{-3 d_{2}}\right), L_{6}=\mathbb{Q}\left(\sqrt{-3 d_{1}}\right)$.

(v) If $d_{1} \equiv 3(\bmod 9)$ and $d_{2} \equiv 2(\bmod 3)$, then $3-\operatorname{rank}\left(K_{2} \mathcal{O}_{F}\right)=3-\operatorname{rank}\left(A_{L_{3}}[1 / 3]\right)+3-\operatorname{rank}\left(A_{L_{5}}\right)+3-\operatorname{rank}\left(A_{L_{6}}\right)+1$, where $L_{3}=\mathbb{Q}\left(\sqrt{-3 d_{1} d_{2}}\right), L_{5}=\mathbb{Q}\left(\sqrt{-3 d_{2}}\right), L_{6}=\mathbb{Q}\left(\sqrt{-3 d_{1}}\right)$.

Proof. (i) By the assumption and Lemma 2.2, we have

$$
A_{L}=A_{L_{1}} \oplus A_{L_{2}} \oplus A_{L_{3}} \oplus A_{L_{4}} \oplus A_{L_{5}} \oplus A_{L_{6}} .
$$

We recall that $\sigma$ is a generator of $\operatorname{Gal}(L / F)$. Then

$$
\sigma^{(p-1) / 2} \varepsilon_{p-2}=\omega\left(g^{(p-1) / 2}\right)^{p-2} \varepsilon_{p-2}=-\varepsilon_{p-2} .
$$

On the other hand, it is easy to check that $\sigma^{(p-1) / 2}$ acts trivially on $A_{L_{1}}$, $A_{L_{2}}$ and $A_{L_{4}}$. Therefore, the result follows from Lemmas 2.1, 3.1 and 3.2.

(ii) By the proof of (i), we have

$$
3-\operatorname{rank}\left(K_{2} \mathcal{O}_{F}\right)=3-\operatorname{rank}\left(\varepsilon_{1} A_{L_{3}}\right)+3-\operatorname{rank}\left(\varepsilon_{1} A_{L_{5}}\right)+3-\operatorname{rank}\left(\varepsilon_{1} A_{L_{6}}\right),
$$

where $L_{3}=\mathbb{Q}\left(\sqrt{-3 d_{1} d_{2}}\right), L_{5}=\mathbb{Q}\left(\sqrt{-3 d_{2}}\right), L_{6}=\mathbb{Q}\left(\sqrt{-3 d_{1}}\right)$. It is easy to see that $\operatorname{Gal}\left(L_{i} / \mathbb{Q}\right)=\langle\sigma\rangle$ for $i=3,5,6$. So $A_{L_{i}}=\varepsilon_{1} A_{L_{i}}$ for $i=3,5,6$, completing the proof.

(iii) By Lemmas 2.1, 2.2 and 3.1, we have

$$
\begin{aligned}
3-\operatorname{rank}\left(K_{2} \mathcal{O}_{F}\right)= & 3-\operatorname{rank}\left(\varepsilon_{1} A_{L_{3}}[1 / 3]\right)+3-\operatorname{rank}\left(\varepsilon_{1} A_{L_{5}}[1 / 3]\right) \\
& +3-\operatorname{rank}\left(\varepsilon_{1} A_{L_{6}}[1 / 3]\right)+2,
\end{aligned}
$$

where $L_{3}=\mathbb{Q}\left(\sqrt{-3 d_{1} d_{2}}\right), L_{5}=\mathbb{Q}\left(\sqrt{-3 d_{2}}\right), L_{6}=\mathbb{Q}\left(\sqrt{-3 d_{1}}\right)$. Since $d_{1} \equiv 3$ $(\bmod 9)$ and $d_{2} \equiv 1(\bmod 3)$, we have $\left(\frac{-d_{1} / 3}{3}\right)=-1$ and $\left(\frac{-d_{1} d_{2} / 3}{3}\right)=-1$. Hence 3 is inert is $L_{3}$ and $L_{6}$. Clearly, 3 is ramified in $L_{5}$. So $A_{L_{i}}[1 / 3]=A_{L_{i}}$, where $i=3,5,6$. Therefore, the result follows from the proof of (ii).

The proofs of (iv) and (v) are similar to that of (iii).

EXAMPLE. As an application of Theorem 3.3, we calculate the 3-rank of $K_{2} \mathcal{O}_{F}$ when $-50<d_{1}, d_{2}<50$. The results are given in the tables of Section 5 . 
Let $\operatorname{Gal}\left(F_{1} / \mathbb{Q}\right)=\left\langle\tau_{1}\right\rangle$ and $\operatorname{Gal}\left(F_{2} / \mathbb{Q}\right)=\left\langle\tau_{2}\right\rangle$. Then $T:=\operatorname{Gal}(F / \mathbb{Q})=$ $\left\langle\tau_{1}, \tau_{2}\right\rangle$. We consider the following idempotents:

$$
\begin{array}{ll}
\theta_{0}=\left(\frac{1+\tau_{1}}{2}\right)\left(\frac{1+\tau_{2}}{2}\right), & \theta_{1}=\left(\frac{1-\tau_{1}}{2}\right)\left(\frac{1+\tau_{2}}{2}\right), \\
\theta_{2}=\left(\frac{1+\tau_{1}}{2}\right)\left(\frac{1-\tau_{2}}{2}\right), & \theta_{3}=\left(\frac{1-\tau_{1}}{2}\right)\left(\frac{1-\tau_{2}}{2}\right) .
\end{array}
$$

Lemma 3.4. For $p=4 s+1$, we have

$$
\begin{aligned}
& \theta_{1} \varepsilon_{2}=\frac{1}{p-1}\left(\frac{1-\tau_{1}}{4}\right) \sum_{k=0}^{s-1} \omega^{2 k}(g) \sigma^{-k} N_{L / E_{1}}, \\
& \theta_{2} \varepsilon_{2}=\frac{1}{p-1}\left(\frac{1-\tau_{2}}{4}\right) \sum_{k=0}^{s-1} \omega^{2 k}(g) \sigma^{-k} N_{L / E_{2}}, \\
& \theta_{3} \varepsilon_{2}=\frac{1}{p-1}\left(\frac{1-\tau_{1}}{4}\right) \sum_{k=0}^{s-1} \omega^{2 k}(g) \sigma^{-k} N_{L / E_{3}},
\end{aligned}
$$

where $E_{1}, E_{2}$ and $E_{3}$ are the subfields of $L$ fixed by $\left\langle\tau_{2}, \sigma^{s} \tau_{1}\right\rangle,\left\langle\tau_{1}, \sigma^{s} \tau_{2}\right\rangle$ and $\left\langle\tau_{1} \tau_{2}, \sigma^{s} \tau_{1}\right\rangle$ respectively.

Proof. Since $\omega^{2 s}(g)=-1$ and $\sigma^{4 s}=1$, we get

$$
\begin{aligned}
\varepsilon_{2} & =\frac{1}{p-1} \sum_{k=0}^{s-1} \omega^{2 k}(g) \sigma^{-k}\left(1+\omega^{2 s}(g) \sigma^{-s}+\omega^{4 s}(g) \sigma^{-2 s}+\omega^{6 s}(g) \sigma^{-3 s}\right) \\
& =\frac{1}{p-1} \sum_{k=0}^{s-1} \omega^{2 k}(g) \sigma^{-k}\left(1-\sigma^{s}+\sigma^{2 s}-\sigma^{3 s}\right) \\
& =\frac{1}{p-1} \sum_{k=0}^{s-1} \omega^{2 k}(g) \sigma^{-k}\left(1-\sigma^{s}\right)\left(1+\sigma^{2 s}\right) .
\end{aligned}
$$

For (1),

$$
\begin{aligned}
\theta_{1} \varepsilon_{2} & =\left(\frac{1-\tau_{1}}{2}\right)\left(\frac{1+\tau_{2}}{2}\right) \frac{1}{p-1} \sum_{k=0}^{s-1} \omega^{2 k}(g) \sigma^{-k}\left(1-\sigma^{s}\right)\left(1+\sigma^{2 s}\right) \\
& =\frac{1}{p-1}\left(\frac{1-\tau_{1}}{4}\right) \sum_{k=0}^{s-1} \omega^{2 k}(g) \sigma^{-k}\left(1+\tau_{2}\right)\left(1+\tau_{1} \sigma^{s}\right)\left(1+\sigma^{2 s}\right) \\
& =\frac{1}{p-1}\left(\frac{1-\tau_{1}}{4}\right) \sum_{k=0}^{s-1} \omega^{2 k}(g) \sigma^{-k} N_{L / E_{1}} .
\end{aligned}
$$

The equalities (2) and (3) can be proved similarly. 
LEMma 3.5 ([2]). $p$-rank $\left(\varepsilon_{k} A_{L}\right)=p$-rank $\left(\varepsilon_{p-k} B_{0}\right)$ for $0 \leq k \leq p$, where we identified $\varepsilon_{p-1}=\varepsilon_{0}$ and $\varepsilon_{p}=\varepsilon_{1}$.

Lemma 3.6. For $0 \leq k \leq 3$ and $0 \leq j \leq p-2$, we have

$$
p-\operatorname{rank}\left(\theta_{k} \varepsilon_{p-j} A_{L}\right) \leq p-\operatorname{rank}\left(\theta_{k} \varepsilon_{j} A_{L}\right)+p-\operatorname{rank}\left(\theta_{k} \varepsilon_{j}\left(U_{L}^{\prime} / U_{L}^{p}\right)\right) .
$$

Proof. Since $\phi: B_{0} \rightarrow\left(A_{L}\right)_{p}$ is a homomorphism of $\Gamma$-modules, we have

$$
\phi: \theta_{k} \varepsilon_{j} B_{0} \rightarrow \theta_{k} \varepsilon_{j}\left(A_{L}\right)_{p} \quad \text { for } 0 \leq k \leq 3,0 \leq j \leq p-2 .
$$

In view of $\operatorname{Ker} \phi \cong U_{L}^{\prime} / U_{L}^{p}$ we get

$$
(\operatorname{Ker} \phi) \cap\left(\theta_{k} \varepsilon_{j} B_{0}\right) \cong \theta_{k} \varepsilon_{j}\left(U_{L}^{\prime} / U_{L}^{p}\right),
$$

and hence

$$
p-\operatorname{rank}\left(\theta_{k} \varepsilon_{j} B_{0}\right) \leq p-\operatorname{rank}\left(\theta_{k} \varepsilon_{j} A_{L}\right)+p-\operatorname{rank}\left(\theta_{k} \varepsilon_{j}\left(U_{L}^{\prime} / U_{L}^{p}\right)\right) .
$$

Therefore the result follows from Lemma 3.5.

THEOREM 3.7. Let $5 \nmid d_{1} d_{2}$. If $F$ is a totally real field, then

$$
5-\operatorname{rank}\left(K_{2} \mathcal{O}_{F}\right) \leq 5-\operatorname{rank}\left(A_{E_{1}}\right)+5-\operatorname{rank}\left(A_{E_{2}}\right)+5-\operatorname{rank}\left(A_{E_{3}}\right)+3,
$$

and if $F$ is a $C M$ field, then

$$
5-\operatorname{rank}\left(K_{2} \mathcal{O}_{F}\right) \leq 5-\operatorname{rank}\left(A_{E_{1}}\right)+5-\operatorname{rank}\left(A_{E_{2}}\right)+5-\operatorname{rank}\left(A_{E_{3}}\right)+1,
$$

where $E_{1}=\mathbb{Q}\left(\sqrt{5 d_{1}}\right), E_{2}=\mathbb{Q}\left(\sqrt{5 d_{2}}\right), E_{3}=\mathbb{Q}\left(\sqrt{5 d_{1} d_{2}}\right)$.

Proof. By Lemmas 2.1, 3.1 and 3.2, 5-rank $\left(K_{2} \mathcal{O}_{F}\right)=5-\operatorname{rank}\left(\varepsilon_{3} A_{L}\right)$. Since $\theta_{0} \varepsilon_{3} A_{L} \subset A_{\mathbb{Q}\left(\zeta_{5}\right)}$, Lemmas 3.4 and 3.6 yield

$$
5-\operatorname{rank}\left(K_{2} \mathcal{O}_{F}\right) \leq \sum_{k=1}^{3}\left(5-\operatorname{rank}\left(A_{E_{k}}\right)+5-\operatorname{rank}\left(U_{E_{k}} / U_{E_{k}}^{5}\right)\right)
$$

By the Dirichlet unit theorem, we conclude that $5-\operatorname{rank}\left(U_{E_{k}} / U_{E_{k}}^{5}\right)=1$ resp. 0 if $E_{k}$ is a real resp. imaginary quadratic field. This completes the proof.

4. $p^{n}$-rank. In this section, we use the same notation as in Section 2.

LEMma 4.1 ([3]). Let $F$ be a number field, and for any odd prime $p$ and a positive integer $n$, let $\Gamma=\operatorname{Gal}\left(F\left(\zeta_{p^{n}}\right) / F\right)$. We have an exact sequence

$(*) \quad 0 \rightarrow\left(\mu_{p^{n}} \otimes C l\left(\mathcal{O}_{F\left(\zeta_{p^{n}}\right)}[1 / p]\right)\right)_{\Gamma} \stackrel{\iota}{\rightarrow} K_{2} \mathcal{O}_{F} / p^{n}$

$$
\stackrel{\lambda}{\rightarrow} \bigoplus_{\mathfrak{p} \mid p}\left(\mu_{p^{n}}\right)_{Z_{\mathfrak{p}}} \stackrel{c}{\rightarrow}\left(\mu_{p^{n}}\right)_{\Gamma} \rightarrow 0 .
$$


Theorem 4.2. Let $F=\mathbb{Q}\left(\sqrt{d_{1}}, \sqrt{d_{2}}\right)$ and $L=F\left(\zeta_{p^{n}}\right)$, where $n \geq 2$.

(i) If $p>3$, then $K_{2} \mathcal{O}_{F} / p^{n} \cong\left(\mu_{p^{n}} \otimes C l\left(\mathcal{O}_{L}[1 / p]\right)\right)_{\Gamma}$.

(ii) $K_{2} \mathcal{O}_{F} / 3^{n} \cong\left(\mu_{3^{n}} \otimes C l\left(\mathcal{O}_{L}[1 / 3]\right)\right)_{\Gamma}$ provided $d_{1}=-3$ and $\left(\frac{d_{2}}{3}\right)=-1$, or $3 \mid d_{1}$ with $\left(\frac{-d_{1} / 3}{3}\right)=-1$ and $\left(\frac{d_{2}}{3}\right)=1$, or $3 \nmid d_{1}$ and $3 \nmid d_{2}$.

(iii) We have an exact sequence

$$
0 \rightarrow\left(\mu_{3^{n}} \otimes C l\left(\mathcal{O}_{L}[1 / 3]\right)\right)_{\Gamma} \rightarrow K_{2} \mathcal{O}_{F} / 3^{n} \rightarrow \mu_{3} \rightarrow 0
$$

provided $d_{1}=-3$ and $\left(\frac{d_{2}}{3}\right)=1$, or $3 \mid d_{1}$ with $\left(\frac{-d_{1} / 3}{3}\right)=1$ and $\left(\frac{d_{2}}{3}\right)=-1$, or $3 \mid d_{1}$ with $\left(\frac{-d_{1} / 3}{3}\right)=-1$ and $\left(\frac{d_{2}}{3}\right)=-1$.

(iv) We have an exact sequence

$$
0 \rightarrow\left(\mu_{3^{n}} \otimes C l\left(\mathcal{O}_{L}[1 / 3]\right)\right)_{\Gamma} \rightarrow K_{2} \mathcal{O}_{F} / 3^{n} \rightarrow \mu_{3} \oplus \mu_{3} \rightarrow 0
$$

provided $3 \mid d_{1}$ with $\left(\frac{-d_{1} / 3}{3}\right)=1$ and $\left(\frac{d_{2}}{3}\right)=1$.

Proof. Since $\mu_{p^{n}}$ is a cohomologically trivial $Z$-module for every subgroup $Z$ of $\Gamma$, we get

$$
\left(\mu_{p^{n}}\right)_{Z_{\mathfrak{p}}} \cong\left(\mu_{p^{n}}\right)^{Z_{\mathfrak{p}}}=\mu_{p^{n}} \cap L^{Z_{\mathfrak{p}}}
$$

where $L^{Z}$ is the decomposition field of $\mathfrak{p}$ in the extension $L / F$. From the conclusions of Section 2, this field is $F$ or $E=\mathbb{Q}\left(\sqrt{d_{1}}, \sqrt{d_{2}}, \sqrt{p^{*}}\right)$. Obviously, the $p$ th root of 1 , where $p>3$, does not belong to this field. So (i) is proved by Lemma 4.1 .

From the conclusions of Section 2, we have the following results:

If $d_{1}=-3$ and $\left(\frac{d_{2}}{3}\right)=1$, then $\bigoplus_{\mathfrak{p} \mid 3}\left(\mu_{3^{n}}\right)_{Z_{\mathfrak{p}}} \cong \mu_{3} \oplus \mu_{3}$ and $\left(\mu_{3^{n}}\right)_{\Gamma} \cong \mu_{3}$.

If $d_{1}=-3$ and $\left(\frac{d_{2}}{3}\right)=-1$, then $\bigoplus_{\mathfrak{p} \mid 3}\left(\mu_{3^{n}}\right)_{Z_{\mathfrak{p}}} \cong \mu_{3}$ and $\left(\mu_{3^{n}}\right)_{\Gamma} \cong \mu_{3}$.

If $3 \mid d_{1} \neq-3,\left(\frac{-d_{1} / 3}{3}\right)=1$ and $\left(\frac{d_{2}}{3}\right)=1$, then $\bigoplus_{\mathfrak{p} \mid 3}\left(\mu_{3^{n}}\right)_{Z_{\mathfrak{p}}} \cong \mu_{3} \oplus \mu_{3}$ and $\left(\mu_{3^{n}}\right)_{\Gamma}=\{1\}$.

If $3 \mid d_{1} \neq-3,\left(\frac{-d_{1} / 3}{3}\right)=1$ and $\left(\frac{d_{2}}{3}\right)=-1$, then $\bigoplus_{\mathfrak{p} \mid 3}\left(\mu_{3^{n}}\right)_{Z_{\mathfrak{p}}} \cong \mu_{3}$ and $\left(\mu_{3^{n}}\right)_{\Gamma}=\{1\}$.

If $3 \mid d_{1} \neq-3,\left(\frac{-d_{1} / 3}{3}\right)=-1$ and $\left(\frac{d_{2}}{3}\right)=1$, then $\bigoplus_{\mathfrak{p} \mid 3}\left(\mu_{3^{n}}\right)_{Z_{\mathfrak{p}}}=\{1\}$ and $\left(\mu_{3^{n}}\right)_{\Gamma}=\{1\}$.

If $3 \mid d_{1} \neq-3,\left(\frac{-d_{1} / 3}{3}\right)=-1$ and $\left(\frac{d_{2}}{3}\right)=-1$, then $\bigoplus_{\mathfrak{p} \mid 3}\left(\mu_{3^{n}}\right)_{Z_{\mathfrak{p}}} \cong \mu_{3}$ and $\left(\mu_{3^{n}}\right)_{\Gamma}=\{1\}$.

If $3 \nmid d_{1}$ and $3 \nmid d_{2}$, then $\bigoplus_{\mathfrak{p} \mid 3}\left(\mu_{3^{n}}\right)_{Z_{\mathfrak{p}}}=\{1\}$ and $\left(\mu_{3^{n}}\right)_{\Gamma}=\{1\}$.

Now (ii)-(iv) follow from Lemma 4.1. 


\section{Tables}

Table 1. $\left(d_{1}, d_{2}\right)$ with $3-\operatorname{rank}\left(K_{2} \mathcal{O}_{F}\right)=1$

\begin{tabular}{|c|c|c|c|c|c|c|c|c|}
\hline$(-47,-43)$ & $(-47,-31)$ & $(-47,-26)$ & $(-47,-22)$ & $(-47,-13)$ & $(-47,-5)$ & $(-47,29)$ & $(-47,43)$ & $(-46,-19)$ \\
\hline$(-46,-17)$ & $(-46,-11)$ & $(-46,-7)$ & $(-46,29)$ & $(-46,43)$ & $(-43,-34)$ & $(-43,-31)$ & $(-43,-26)$ & $(-43,-17)$ \\
\hline$(-43,-11)$ & $(-43,-1)$ & $(-43,14)$ & $(-43,29)$ & $(-41,-17)$ & $(-41,-14)$ & $(-41,-13)$ & $(-41,-10)$ & $(-41,-7)$ \\
\hline$(-41,-2)$ & $(-41,13)$ & $(-41,29)$ & $(-41,43)$ & $(-38,-37)$ & $(-38,-35)$ & $(-38,-29)$ & $(-38,-23)$ & $(-38,-13)$ \\
\hline$(-38,-11)$ & $(-38,29)$ & $(-38,43)$ & $(-37,-35)$ & $(-37,-34)$ & $(-37,-23)$ & $(-37,-14)$ & $(-37,-2)$ & $(-37,29)$ \\
\hline$-37,43)$ & $(-35,29)$ & $(-35,43)$ & $(-34,-23)$ & $(-34,-7)$ & $(-34,29)$ & $(-34,43)$ & $(-31,-29)$ & $(-31,-26)$ \\
\hline$-31,-10)$ & $(-31,-2)$ & $(-31,23)$ & $(-31,29)$ & $(-31,43)$ & $(-29,-26)$ & $(-29,-23)$ & $(-29,-22)$ & $(-29,-14)$ \\
\hline$-29,-2)$ & $(-29,-1)$ & $(-29,43)$ & $(-26,-19)$ & $(-26,-7)$ & $(-26,29)$ & $(-26,43)$ & $(-23,-22)$ & $(-23,-17)$ \\
\hline$(-23,-14)$ & $(-23,-11)$ & $(-23,29)$ & $(-23,31)$ & $(-23,43)$ & $(-22,-19)$ & $(-22,29)$ & $(-22,43)$ & $(-19,-17)$ \\
\hline$-19,-1$ & $(-19,29)$ & $(-19,43)$ & $(-17,-14)$ & $(-17,-5)$ & $(-17,29)$ & $(-17,43)$ & $(-14,-13)$ & $(-14,29)$ \\
\hline$-13,29)$ & $(-13,41)$ & $(-13,43)$ & $(-11,-7)$ & $(-11,29)$ & $(-11,43)$ & $(-10,29)$ & $(-10,43)$ & $(-7,29)$ \\
\hline 3) & $-5,2$ & $(-5$, & $(-2,29)$ & $(-2,43)$ & $(-1$ & & & \\
\hline 41) & $(2,43)$ & $(5,17)$ & $(5,29)$ & $(5,43)$ & $(5,47)$ & $(7,1$ & $(7$, & $(7,29)$ \\
\hline 7,34$)$ & $(7,41)$ & $(7,4$ & $(7,4$ & $(10$ & 0 & $(10$, & $(10$ & 3) \\
\hline 1. & 1 & 1 & ) & ) & ) & ( & ) & ) \\
\hline 3,47$)$ & $4,17)$ & $4,37)$ & 4, & $(14$, & $(17,19)$ & $(17$, & $(17$, & $(17,46)$ \\
\hline ) & $9,26)$ & $(19$, & 9, & $9,46)$ & ) & $(22$, & $(22$, & (23, \\
\hline 23,37$)$ & $(23,38)$ & $(23,43)$ & $(26,31)$ & $(26,43)$ & $(26,47)$ & $(29,34)$ & $(29$, & $(29,37)$ \\
\hline 29,3 & $(29,41)$ & $(29,4$ & $9,47)$ & 34 & $(35$, & $(35$, & $(35$, & $(35$, \\
\hline 7,38$)$ & $(37,43)$ & $(38,43)$ & $(41$, & $(43,46)$ & $(-39$, & $(-39,-2)$ & $(-39$ & $(-30,-$ \\
\hline$(-30,-11)$ & $-30,43)$ & $(-21,4$ & $(6,13)$ & $(6,19)$ & (6, & $(15$, & $(15$ & $(15$ \\
\hline$(-47,-21)$ & $(-47$ & $(-41$, & $(-41$ & $(-26,-$ & $(10$, & $(13$ & (31. & $(-4$ \\
\hline$(-42,-31)$ & $(-42,-19)$ & $(-42,-13)$ & $(-42,-1)$ & $(-42,5)$ & $(-42,11)$ & $(-42,17)$ & $(-42,23)$ & $(-42,41)$ \\
\hline$(-42,47)$ & $(-33,-31)$ & $(-33,-19)$ & $(-33,-13)$ & $(-33,-10)$ & $(-33,-7)$ & $(-33,-1)$ & $(-33$ & $(-3$ \\
\hline$-33,14)$ & $(-33,17)$ & $(-33,23)$ & $(-33,26)$ & $(-33,3$ & $(-33,38)$ & $(-33,41)$ & $(-33,47)$ & $(-15,-1$ \\
\hline$(-15,-7)$ & $(-15,-1)$ & $(-15,2)$ & $(-15,11)$ & $(-15,14)$ & $(-15,17)$ & $(-15,23)$ & $(-15,26)$ & $(-15,38)$ \\
\hline$(-15,41)$ & $(-15,47)$ & $(-6,-1)$ & $(-6,5)$ & $(-6,11)$ & $(-6,17)$ & $(-6,23$ & $(-6$, & $(-6$ \\
\hline$-6,47)$ & $(3,5)$ & 3,1 & (3. & $(3,17)$ & $(3,23)$ & $(3,26)$ & $(3$, & $(3$, \\
\hline 3,41$)$ & $(3,47)$ & $(21$, & $(21$, & $(21$, & (21, & $(21$, & $(30$ & $(30$ \\
\hline 39,41$)$ & $(39,47)$ & $(-46,-$ & $(-46,-15)$ & $(-46,3)$ & $(-46$ & $(-46$ & $(-43,-$ & $(-43,-33)$ \\
\hline$(-43,-15)$ & $(-43,-6)$ & $(-43,3)$ & $(-43,21)$ & $(-43,30)$ & $(-43,39)$ & $(-37,-33)$ & $(-37,-15)$ & $(-37,-6)$ \\
\hline$(-37,3)$ & $(-37,21)$ & -37, & $(-37,3$ & $(-34,-$ & $(-34,-$ & $(-3$ & $(-$ & $(-$ \\
\hline$(-31,-15)$ & $(-31,-6)$ & $(-31,3)$ & $(-31,21)$ & $(-31,30)$ & $(-31,39)$ & $(-22,-15)$ & $(-22,3)$ & $(-22,21)$ \\
\hline$(-22,39)$ & $(-19,-15)$ & $(-19,-6)$ & $(-19,3)$ & $(-19,21)$ & $(-19,30)$ & $(-19,35$ & $(-13,-$ & $(-1$ \\
\hline$(-13,21)$ & $(-13,30)$ & $(-10,3)$ & $(-10,21)$ & $(-10$ & $(-7,-6)$ & $(-7,3)$ & $(-7,30)$ & $(-7,39)$ \\
\hline$-1,3$ & $(-1,2$ & $(-1,3$ & $(-1,39)$ & $(2,3)$ & $(2,21)$ & $(2$, & $(5$, & $(5,3$ \\
\hline 11,21 & $(11,30)$ & $(11$, & $(14$, & $(17$, & $(17$, & $(17$ & $(2$ & \\
\hline$(35,39)$ & $(38,39)$ & $(-39,-37)$ & $(-39,-34)$ & $(-39,-31)$ & $(-39,-10)$ & $(-39,-7)$ & $(-39,-1)$ & $(-39,2)$ \\
\hline$-39,5$ & $(-39$ & -39 & $(-39,1$ & $(-39$ & $(-39$, & $(-3$ & $(-3$ & $(-3$ \\
\hline$(-30,-19)$ & $(-30,-13)$ & $(-30,-7)$ & $(-30,-1)$ & $(-30,11)$ & $(-30,17)$ & $(-30,23)$ & $(-30,41)$ & $(-30,47)$ \\
\hline$(-21,-19)$ & $(-21,-13)$ & $(-21,-10)$ & $(-21,-1)$ & $(-21,2)$ & $(-21,5)$ & $(-2)$ & $(-$ & $(-$ \\
\hline$(-21,26)$ & $(-21,38)$ & $(-21,41)$ & $(-21,47)$ & $(6,11)$ & $(6,17)$ & $(6,23)$ & $(6,35)$ & $(6,41)$ \\
\hline$(6,47)$ & $(15,17)$ & $(15,23)$ & $(15,26)$ & $(15,38)$ & $(15,41)$ & $(15,47)$ & $(33,35)$ & $(33,38)$ \\
\hline$(42,47)$ & $(-46,-39)$ & $(-46,-21)$ & $(-46,15)$ & $(-43,-39)$ & $(-43,-21)$ & $(-43,6)$ & $(-43,15)$ & $(-43,42)$ \\
\hline$(-37,-21)$ & $(-37,6)$ & $(-37,15)$ & $(-37,33)$ & $(-37,42)$ & $(-34,-21)$ & $(-34,15)$ & $(-34,33)$ & $(-31,-30)$ \\
\hline$(-31,-21)$ & $(-31,6)$ & $(-31,15)$ & $(-31,33)$ & $(-31,42)$ & $(-22,-21)$ & $(-22,15)$ & $(-19,6)$ & $(-19,15)$ \\
\hline$(-19,33)$ & $(-19,42)$ & $(-13,6)$ & $(-13,15)$ & $(-13,33)$ & $(-13,42)$ & $(-10,33)$ & $(-7,6)$ & $(-7,15)$ \\
\hline$(-7,3$ & $(-1,6)$ & $(-1,15)$ & $(-1,3$ & $(-1,42)$ & $(2,15)$ & $(2,33)$ & $(5,6)$ & $(5,33)$ \\
\hline$(5,42)$ & $(11,15)$ & $(11,42)$ & $(14,15)$ & $(14,33)$ & $(17,33)$ & $(17,42)$ & $(23,33)$ & $(23,42)$ \\
\hline & & & & & & & & \\
\hline
\end{tabular}


Table 2. $\left(d_{1}, d_{2}\right)$ with $3-\operatorname{rank}\left(K_{2} \mathcal{O}_{F}\right)=2$

\begin{tabular}{lllllllll}
\hline$(-14,43)$ & $(2,29)$ & $(11,43)$ & $(14,29)$ & $(17,43)$ & $(22,29)$ & $(23,29)$ & $(26,29)$ & $(29,31)$ \\
$(29,43)$ & $(31,43)$ & $(34,43)$ & $(43,47)$ & $(-42,-41)$ & $(-42,-29)$ & $(-42,-23)$ & $(-42,-17)$ & $(-42,-11)$ \\
$(-42,-5)$ & $(-42,1)$ & $(-42,13)$ & $(-42,19)$ & $(-42,31)$ & $(-42,37)$ & $(-33,-29)$ & $(-33,-23)$ & $(-33,-17)$ \\
$(-33,-14)$ & $(-33,-5)$ & $(-33,-2)$ & $(-33,1)$ & $(-33,7)$ & $(-33,10)$ & $(-33,13)$ & $(-33,19)$ & $(-33,31)$ \\
$(-33,34)$ & $(-33,37)$ & $(-15,-14)$ & $(-15,-11)$ & $(-15,-2)$ & $(-15,1)$ & $(-15,7)$ & $(-15,13)$ & $(-15,19)$ \\
$(-15,22)$ & $(-15,31)$ & $(-15,34)$ & $(-15,37)$ & $(-15,46)$ & $(-6,-5)$ & $(-6,1)$ & $(-6,7)$ & $(-6,13)$ \\
$(-6,19)$ & $(-6,31)$ & $(-6,37)$ & $(-6,43)$ & $(3,7)$ & $(3,10)$ & $(3,13)$ & $(3,19)$ & $(3,22)$ \\
$(3,34)$ & $(3,37)$ & $(3,46)$ & $(21,22)$ & $(21,31)$ & $(21,34)$ & $(21,37)$ & $(21,46)$ & $(30,31)$ \\
$(39,46)$ & $(-47,-42)$ & $(-47,-15)$ & $(-47,-6)$ & $(-47,3)$ & $(-47,21)$ & $(-47,30)$ & $(-47,39)$ & $(-41,-15)$ \\
$(-41,-6)$ & $(-41,3)$ & $(-41,21)$ & $(-41,30)$ & $(-41,39)$ & $(-38,-33)$ & $(-38,-15)$ & $(-38,3)$ & $(-38,21)$ \\
$(-38,39)$ & $(-35,-33)$ & $(-35,-6)$ & $(-35,3)$ & $(-35,39)$ & $(-29,-15)$ & $(-29,-6)$ & $(-29,3)$ & $(-29,21)$ \\
$(-29,30)$ & $(-29,39)$ & $(-26,-15)$ & $(-26,3)$ & $(-26,21)$ & $(-23,-15)$ & $(-23,-6)$ & $(-23,3)$ & $(-23,21)$ \\
$(-23,30)$ & $(-23,39)$ & $(-17,-15)$ & $(-17,-6)$ & $(-17,3)$ & $(-17,21)$ & $(-17,30)$ & $(-17,39)$ & $(-14,3)$ \\
$(-14,39)$ & $(-11,-6)$ & $(-11,3)$ & $(-11,21)$ & $(-11,30)$ & $(-11,39)$ & $(-5,3)$ & $(-5,21)$ & $(-5,39)$ \\
$(-2,3)$ & $(-2,21)$ & $(-2,39)$ & $(1,3)$ & $(1,21)$ & $(1,30)$ & $(1,39)$ & $(7,30)$ & $(7,39)$ \\
$(10,21)$ & $(10,39)$ & $(13,21)$ & $(13,30)$ & $(19,21)$ & $(19,30)$ & $(31,39)$ & $(34,39)$ & $(37,39)$ \\
$(33,43)$ & $(42,43)$ & $(-42,29)$ & $(-33,29)$ & $(-15,29)$ & $(-6,29)$ & $(3,29)$ & $(21,29)$ & $(29,30)$ \\
$(29,39)$ & $(-39,-22)$ & $(-39,-19)$ & $(-39,29)$ & $(-30,29)$ & $(-21,29)$ & $(6,29)$ & $(15,29)$ & $(33,41)$ \\
$(33,47)$ & $(-46,33)$ & $(-43,-30)$ & $(-43,33)$ & $(-37,-30)$ & $(26,33)$ & $(29,33)$ & $(29,42)$ & \\
\hline
\end{tabular}

Table 3. $\left(d_{1}, d_{2}\right)$ with $3-\operatorname{rank} K_{2} \mathcal{O}_{F}=3$

\begin{tabular}{|c|c|c|c|c|c|c|c|}
\hline$(-42,43)$ & $(-33,-47)$ & $(-33,-41)$ & $(-33,-26)$ & $(-33,46) \quad(-15,43)$ & $(-6,43)$ & $(3,31)$ & $(3,43)$ \\
\hline$(21,43)$ & $(30,37)$ & $(39,19)$ & $(39,22)$ & $(39,43)$ & & & \\
\hline
\end{tabular}

Finally, there are only two pairs $(-33,43)$ and $(30,43)$ with $3-\operatorname{rank}\left(K_{2} \mathcal{O}_{F}\right)=4$. For any $\left(d_{1}, d_{2}\right)$ which does not appear in the above tables, the 3-primary part of $K_{2} \mathcal{O}_{F}$ is trivial.

Acknowledgments. I would like to thank the referee for many valuable suggestions. This paper was supported by NSFC 10801076, NSFC 10971098 and the Natural Science Foundation of the Jiangsu Higher Education Institutions of China (Grant No. 08KJB110006).

\section{References}

[1] J. Browkin, Tame kernels of cubic cyclic fields, Math. Comp. 74 (2005), 967-999.

[2] - On the p-rank of the tame kernel of algebraic number fields, J. Reine Angew. Math. 432 (1992), 135-149.

[3] $\mathrm{F}$. Keune, On the structure of $K_{2}$ of the ring of integers in a number field, K-Theory 2 (1989), 625-645.

[4] M. Kolster, Odd torsion in the tame kernel of totally real number fields, in: Algebraic K-Theory: Connections with Geometry and Topology, J. F. Jardine and V. P. Snaith (eds.), Springer, 1989, 177-188.

[5] H. R. Qin, The 2-Sylow subgroups of the tame kernel of imaginary quadratic fields, Acta Arith. 69 (1995), 153-169.

[6] - , The 4-rank of $K_{2} \mathcal{O}_{F}$ for real quadratic fields, ibid. 72 (1995), 323-333.

[7] - , The structure of the tame kernels of quadratic number fields (I), ibid. 113 (2004), 203-240. 
[8] H. R. Qin, The 2-Sylow subgroup of $K_{2} \mathcal{O}_{F}$ for number fields $F$, J. Algebra 284 (2005), 494-519.

[9] —, Tame kernels and Tate kernels of quadratic number fields, J. Reine Angew. Math. 530 (2001), 105-144.

[10] J. Tate, Relations between $K_{2}$ and Galois cohomology, Invent. Math. 36 (1976), $257-274$.

Haiyan Zhou

School of Mathematical Sciences

Nanjing Normal University

Nanjing 210093, P.R. China

E-mail: haiyanxiaodong@gmail.com

Received on 11.9.2009

and in revised form on 3.2.2010 